\title{
Conf-94/182--3
}

GA-A21926

\section{DEVELOPMENT OF A TOKAMAK PLASMA OPTIMIZED FOR STABILITY AND CONFINEMENT}

\author{
by \\ P.A. POLITZER for The.DIII-D TEAM
}

This is a preprint of an invited paper presented at the 6th International Conference on Plasma Physics and Controlled Nuclear Fusion, November 29 through December 2, 1994, in Toki, Japan, and to be printed in the PROCEEDINGS.

\author{
Work supported by \\ the U.S. Department of Energy \\ under Contract Nos. DE-AC03-89ER51114, W-7405-ENG-48, \\ and DE-AC05-840R21400
}

GA PROJECT 3466

FEBRUARY 1995

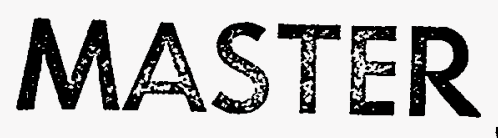




\section{DISCLAIMER}

This report was prepared as an account of work sponsored by an agency of the United States Government. Neither the United States Government nor any agency thereof, nor any of their employees, makes any warranty, express or implied, or assumes any legal liability or responsibility for the accuracy, completeness, or usefulness of any information, apparatus, produce, or process disclosed, or represents that its use would not infringe privately owned rights. Reference herein to any specific commercial product, process, or service by trade name, trademark, manufacturer, or otherwise, does not necessarily constitute or imply its endorsement, recommendation, or favoring by the United States Government or any agency thereof. The views and opinions of authors expressed herein do not necessarily state or reflect those of the United States Government or any agency thereof. 


\section{DISCLAIMER}

Portions of this document may be illegible in electronic image products. Images are produced from the best available original document. 


\title{
DEVELOPMENT OF A TOKAMAK PLASMA OPTIMIZED FOR STABILITY AND CONFINEMENT
}

\author{
P. A. Politzer for the DIII-D Team \\ General Atomics \\ P.O. Box 85608 \\ San Diego, California 92186-9784 \\ (619) $455-2260$
}

\section{ABSTRACT}

Design of an economically attractive tokamak fusion reactor depends on producing steady-state plasma operation with simultaneous high energy density $(\beta)$ and high energy confinement $\left(\tau_{E}\right)$; either of these, by itself, is insufficient. In operation of the DIII-D tokamak, both high confinement enhancement $\left(H \equiv \tau_{\mathrm{E}} / \tau_{\mathrm{ITER}}-89 \mathrm{P}=4\right)$ and high normalized $\beta$ ( $\beta_{\mathrm{N}} \equiv \beta /(\mathrm{V} / \mathrm{aB})=6 \%$-m-T/MA) have been obtained. For the present, these conditions have been produced separately and in transient discharges. The DIII-D advanced tokamak development program is directed toward developing an understanding of the characteristics which lead to high stability and confinement, and to use that understanding to demonstrate stationary, high performance operation through active control of the plasma shape and profiles.

We have identified some of the features of the operating modes in DIII-D that contribute to better performance. These are control of the plasma shape, control of both bulk plasma rotation and shear in the rotation and $E_{r}$ profiles, and particularly control of the toroidal current profiles. In order to guide our future experiments, we are developing optimized scenarios based on our anticipated plasma control capabilities, particularly using fast wave current drive (on-axis) and electron cyclotron current drive (off-axis). The most highly developed model is the second-stable core $\mathrm{VH}$-mode, which has a reversed magnetic shear safety factor profile $\left[q(0)=3.9, q_{\min }=\right.$ 2.6 , and $\left.q_{95}=6\right]$. This model plasma uses profiles which we expect to be realizable. At $\beta_{N} \geq 6$, it is stable to $n=1$ kink modes and ideal ballooning modes, and is expected to reach $\mathrm{H} \geq 3$ with $\mathrm{VH}$-mode-like confinement.

\section{INTRODUCTION}

The advanced tokamak program is an effort to enhance the technical and economic acceptability of the tokamak fusion reactor by improving the performance of the tokamak plasma through the use of plasma profile control techniques. Control of the profiles, particularly of the toroidal plasma current, can improve the performance of the plasma in three respects: increasing the attainable plasma pressure by raising the stability limits, increasing the margin for ignition by raising the energy confinement time, and reducing the need for external power by raising the fraction of current provided by the bootstrap effect. Without these improvements, conceptual designs of tokamak reactors show that large devices are required, with high magnetic field and current. Simultaneously improving the confinement and stability leads to significant reduction in the size and cost of the reactor core, and in the electricity costs. Reduction in the recirculating power with steady-state, high bootstrap fraction operation reduces the overall cost and the unit cost of electricity. Estimates are that steady-state operation with $\beta_{\mathrm{N}} \approx 5-6, \mathrm{H} \approx 3-4, \mathrm{f}_{\mathrm{bs}} \approx$ $0.7-0.8$, at $q \approx 5^{\mathrm{a}}$ may provide a factor of $\approx 40 \%$ reduction in the cost of the tokamak core in a reactor.., 1 Successful achievement of advanced tokamak operation will also allow steady-state operation of ITER.

There has been ample experimental evidence in many plasma confinement devices confirming the existence of improved operating modes. ${ }^{2}$ High values of $\beta_{N}, H$, and $f_{b s}$ are attained under a variety of conditions. However, these high values are not reached simultaneously, and only under transient conditions. The challenge for the tokamak experimental program in the next few years is to

\footnotetext{
${ }^{a} \beta_{N} \equiv 10^{8} \beta /(\mathrm{I} / \mathrm{aB})=80 \pi\langle\mathrm{p}\rangle \mathrm{a} / \mathrm{IB} ; \mathrm{H} \equiv \tau_{\mathrm{E}} / \tau_{\mathrm{ITER}-89 \mathrm{P}} \mathrm{f}_{\mathrm{bs}} \equiv \mathrm{I}_{\mathrm{bs}} / \mathrm{I}$.

${ }^{b}$ Some advantages of advanced tokamak operation can be illustrated by the following simple example: consider a reactor with fixed magnetic field and plasma pressure (i.e., fixed $\beta$ ). In the standard case $q=3, \beta_{N}=3, H=1.8$, and $f_{b s}=0.3$. If advanced tokamak parameters can be achieved, the same plasma is maintained at $q=5$ (with the current reduced by $40 \%$, greatly reducing disruptivity), $\beta_{N}=5, H=3$, and $f_{b s}=0.8$ (reducing the power required for current drive by $85 \%$ ).
} 
show that significant improvements can be achieved simultaneously, and under stationary conditions.

In DIII-D, high values of $\beta_{N}, H$, and $f_{b s}$ have been reached in a variety of operating modes: $\mathrm{VH}$-mode, ${ }^{3}$ high internal inductance (high $\ell_{\mathfrak{j}}$ ) $\mathrm{H}$-mode, ${ }^{4}$ high $\beta_{\mathrm{p}}$ operation, ${ }^{5}$ and second-stable core operation. ${ }^{6}$ The strategy of the DIII-D advanced tokamak program is to identify those characteristics of plasmas in these operating regimes which are associated with improved performance, and to develop and work toward demonstration of scenarios which credibly combine the best features.

In the following sections, we review the characteristics associated with improvement in performance in the categories of strong plasma shaping, plasma rotation and rotation shear, and current and $q$ profile effects. In each of these areas we also note some of the important questions that need to be addressed for development of advanced tokamak reactor scenarios. We then look a particular scenario, the combination of second stability regime operation in the plasma core with VH-mode confinement, and develop modeling and simulation which indicates that this regime can be produced and sustained in DIII-D.

\section{FACTORS AFFECTING PERFORMANCE IMPROVEMENT - EXPERIMENTAL EVIDENCE}

We have found that there are many parameters which influence stability and confinement in the DIII-D tokamak. Among these are the plasma shape, elongation and triangularity; the effects of radial electric fields, rotation, and shear in the plasma flows; the interaction between the rotating plasma and the resistive wall; and the details of the current and $q$ profiles: the internal inductance $\left(\ell_{i}\right)$, the value of $q(0)$, negative shear regions, and currents near the plasma boundary. The pressure profile is not included in this list because we want to restrict these considerations to those parameters which are amenable to external control, even in a reactor. In a fusion reactor, the fusion-generated $\alpha$-particles provide the power source that determines the pressure profile, and no plausible method has yet been identified for direct external control of this profile.

\section{A. Plasma Shape}

The dependence of confinement on plasma shape has recently been explored in a series of experiments in which we systematically studied the variation with elongation, triangularity, and separatrix topology (single- and doublenull). ${ }^{7}$ There is a clear dependence of both $\beta$ and $\tau_{E}$ on triangularity $(\delta)$ in ELM-free states (both $\mathrm{H}-$ and VH- mode). In fact, VH-mode is not obtained for $\delta<0.3$. In ELMing $\mathrm{H}$-mode, the dependence is weak, but in the direction of improvement with increasing $\delta$. The improvement in performance seen in double-null divertor configurations (versus single-null) is accounted for by the higher triangularity possible with a double-null separatrix. Another benefit of high triangularity is that the confinement improvement extends to higher $\beta$ : confinement begins to deteriorate above $\beta_{\mathrm{N}} \approx 2$ for low $\delta$, but only above $\beta_{\mathrm{N}} \approx 4$ at high $\delta$. The dependence of performance on elongation is due primarily to the capability of operation with higher plasma current (for fixed q) at higher $\kappa$.

Measuring performance by the product $\beta \tau_{E}$, we find that, in ELM-free VH-mode, $\beta \tau_{\mathrm{E}}$ is maximum for $q \approx$ $4-4.5$, whereas $\beta_{\mathrm{N}} \mathrm{H}$ peaks for $\mathrm{q} \approx 5-5.5$. $\beta \tau_{\mathrm{E}}$ increases with elongation, but variation of $\beta_{\mathrm{NH}}$ with $\alpha$ is weak. The dependence of performance on shape in $\mathrm{VH}$-mode can be summarized by plotting $\beta \tau_{E}$ vs. $S^{2} R^{2} /\left(1+\kappa^{2}\right)$ (Fig. 1). ${ }^{\mathrm{C}}$

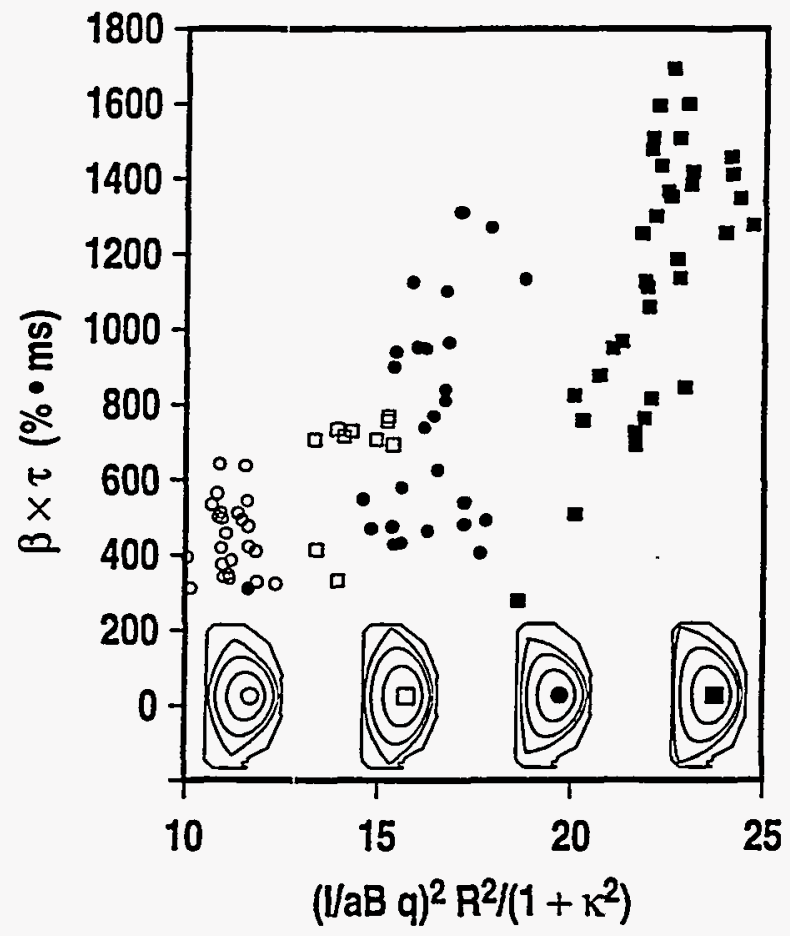

Fig. 1. Plasma performance, measure by $\beta \tau$, improves with increasing plasma shaping. Solid symbols: $\delta=0.9$, open symbols: $\delta=0.3$; circles: $\kappa=1.7$, squares: $\kappa=2.1$.

${ }^{c} S \equiv\left(10^{-6} \mathrm{l} / \mathrm{aB}\right) \mathrm{q} ; \mathrm{S}$ represents a measure of the toroidal current in the plasma for a given value of the safety factor. 


\section{B. Rotation - Internal Transport Barriers - H- and VH-Mode}

The critical importance of plasma rotation in determining stability and transport has been recognized in recent years. One effect of plasma rotation is the combination of rotation, rotation shear, and the radial electric field which leads to the development of the $\mathrm{H}$-mode transport barrier, and possibly also to the VH-mode barrier.

The $\mathrm{H}$-mode is results from development of a transport barrier near the plasma edge. ${ }^{8}$ The paradigm is that (a) in L-mode the edge region has low $\mathrm{E}_{\mathrm{r}}$ shear, high turbulence, large transport, and a relatively shallow pressure gradient; (b) in $\mathrm{H}$-mode the edge has high $\mathrm{E}_{\mathrm{r}}$ shear (the second derivative of $E_{r}$ is also important in some models), low turbulence, low transport, and a steep pressure gradient (limited eventually by relaxation oscillation: ELMs). The development of the $E_{r}$ shear layer occurs very near the edge of the plasma (Fig. 2), possibly associated with development of charge separation due to rapid variation of particle orbits and collisionality with radius across the last closed flux surface. Some of the questions to be addressed in development of advanced tokamak reactor plasmas are: is the steep edge pressure gradient a necessary consequence of $\mathrm{H}$-mode (it leads to a large bootstrap current density at the edge, affecting stability and limiting

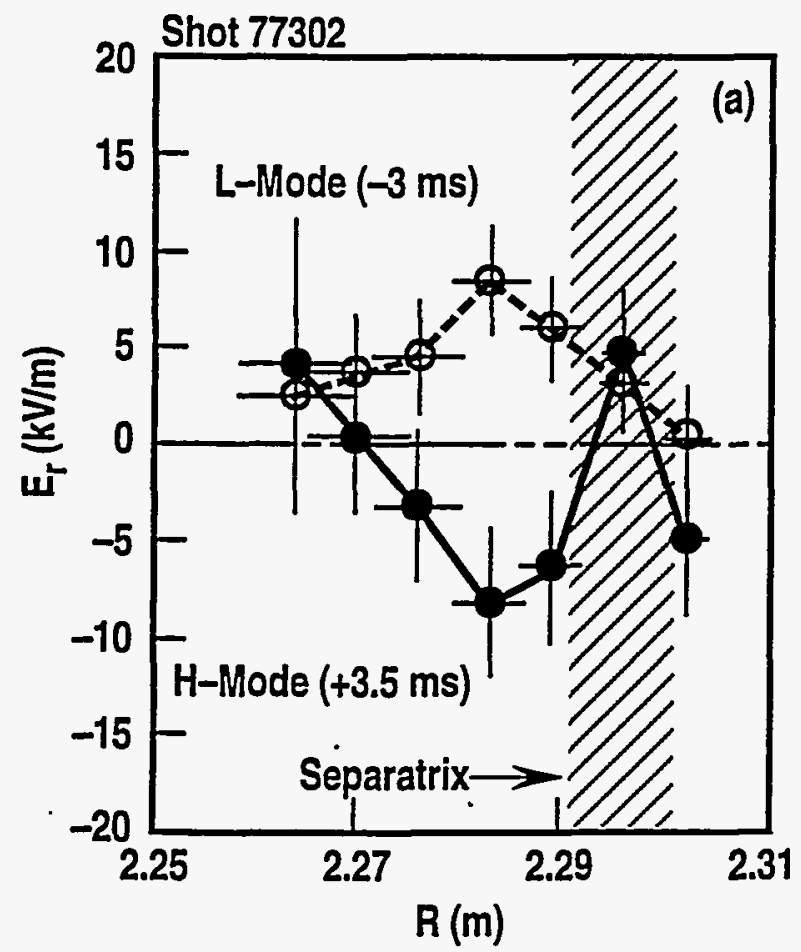

the internal inductance)? Also, are ELMs a necessary consequence of $\mathrm{H}$-mode? Can ELMing be made consistent with improved internal confinement and stability modes (e.g., VH-mode)? Finally, are ELMs necessary for steady-state impurity control?

The VH-mode is clearly associated with the development of a rotation and $E_{\mathrm{r}}$ shear region further into the plasma $(\rho \approx 0.6-0.9)$. The improvement in confinement associated with VH-mode in DIII-D occurs after the $\mathrm{L}-\mathrm{H}$ transition. ${ }^{9}$ One signature of the VH-mode is the toroidal spin-up of the core of the plasma, associated with the development of an $E_{\mathrm{r}}$ and rotation shear layer, generally near $\rho \sim 0.7$, and the reduction of the thermal diffusivity (Fig. 3). The energy confinement time continues to increase during $\mathrm{VH}$-mode, until the $\mathrm{VH}$ phase terminates with a global MHD instability and a transition to ELMing $\mathrm{H}$-mode (Fig. 4). The VH-mode can be extended by feedback controlling input power to hold stored energy constant and stay below instability threshold. Can this be done indefinitely? The VH-mode produces the best transient performance seen in DIII-D, as measured by $\mathrm{nT} \tau$ (up to $\mathrm{nD}_{\mathrm{D}}(0) \mathrm{T}_{\mathrm{i}}(0) \tau_{\mathrm{E}} \approx 5 \times 10^{20} \mathrm{~m}^{-3} \mathrm{keV} \mathrm{s}$ ). ${ }^{10}$

The close tie between rotation and improved confinement is confirmed experiments in which the rotation is reduced by application of a static error field. ${ }^{11}$ With magnetic braking, the extent and magnitude of the $E_{r}$

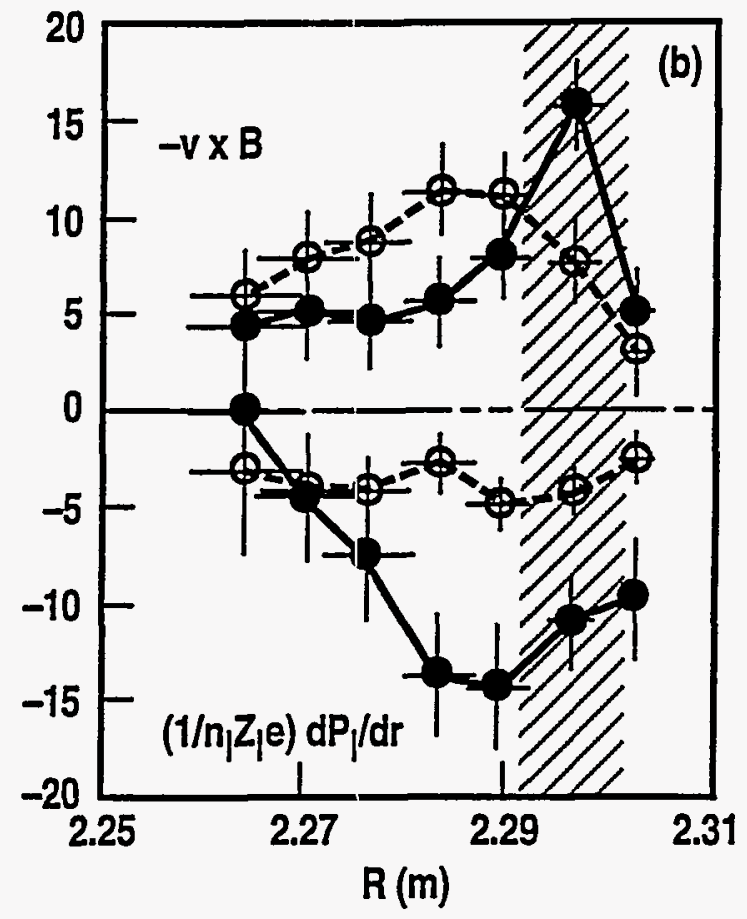

Fig. 2. (a) The radial electric field, and (b) the $\mathrm{v} \times \mathrm{B}$ and pressure gradient terms in the ion force balance, just before (open circles) and after (solid circles) the $\mathrm{L}-\mathrm{H}$ transition, showing the development of the $\mathrm{E}_{\mathrm{r}}$ shear and curvature layer and the increase in $\nabla \mathrm{p}$. 


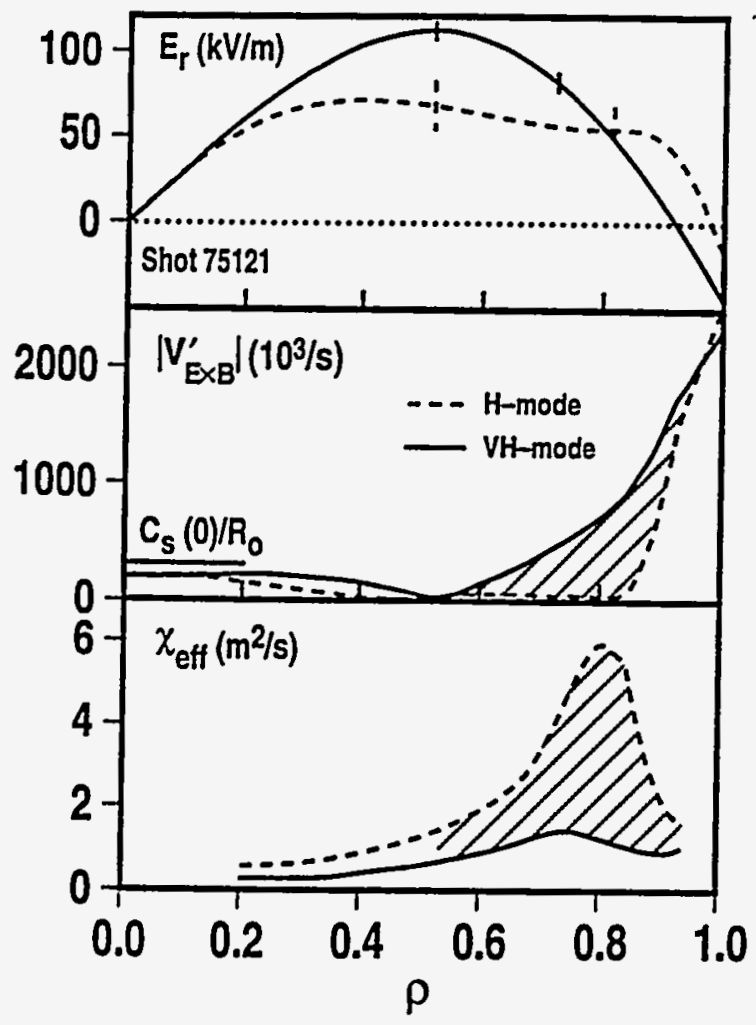

Fig. 3. Profile comparisons between $\mathrm{H}$-mode (dashed lines) and VH-mode (solid lines). After the $\mathrm{H}-\mathrm{VH}$ transition, in the region $0.6 \leq \rho \leq 0.9$, the $V_{E \times B}$ shear increases and the effective thermal diffusivity is reduced.

shear is reduced and the thermal diffusivity increases in the same region. Experiments show similar results in $\mathrm{VH}$-mode and in high $\ell_{\mathbf{i}} \mathrm{H}$-mode.

\section{Rotation - Interaction with Resistive Wall - Kink Stability and Locked Modes}

The other important effect of plasma rotation is the interaction between the rotating plasma and the surrounding resistive wall which leads to changes in the stability of external, long wavelength (low $\mathrm{n}$ ) modes, and in the phenomenon of locked modes.

Recent calculations and experiments have shown clearly that, with plasma rotation, a resistive wall improves to the low $\mathrm{n}$ stability of the plasma. ${ }^{12} \mathrm{High} \beta_{\mathrm{N}}$, $>30 \%$ above the limit calculated for no wall, can be maintained as long as the plasma rotates. In the example shown, when the rotation frequency goes to zero at the $q=3$ surface, a very low frequency $m / n=3 / 1$ instability occurs and the plasma pressure falls (Fig. 5). ${ }^{13}$ This instability is consistent with the theoretically expected resistive wall mode. The reason for the decrease in rotation frequency is presently under investigation. One
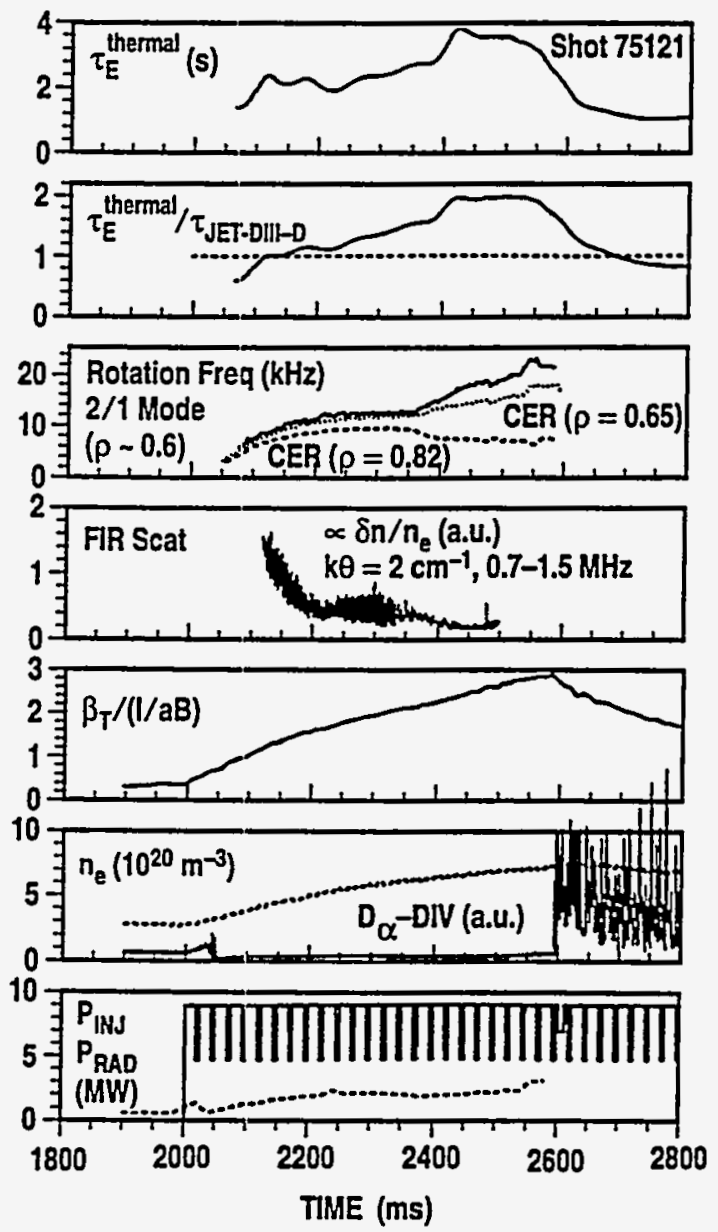

Fig. 4. Characteristic waveforms for a VH-mode discharge. At about $2350 \mathrm{~ms}$, the rate of rise of $\tau_{\mathrm{E}}$ increases, the rotation frequency increases in the plasma core (and decreases near the edge), and the fluctuation level as seen by FIR scattering in the region of spin-up decreases. The VH-mode termninates at about $2590 \mathrm{~ms}$, with a transition to ELMing $\mathrm{H}$-mode.

possibility is that the momentum input may be reduced due to the interaction between the fast ions and Alfvén eigenmode instabilities. Experiments using magnetic braking indicate that only a modest rotation rate $(\geq 1 \mathrm{kHz})$ at the $q=2$ and 3 surfaces may be sufficient for stabilization. 14

Locked modes occur when a plasma instability locks to a stationary nonaxisymmetric magnetic field perturbation (inherent in any real coil system). If the real frequency goes to zero, the mode amplitude grows rapidly and it causes severe loss of confinement. Avoidance of locked modes is done in part through minimizing the static magnetic error fields (through good design and with error-correcting coils), and by maintaining the rotation of the plasma against the drag of the static field. 

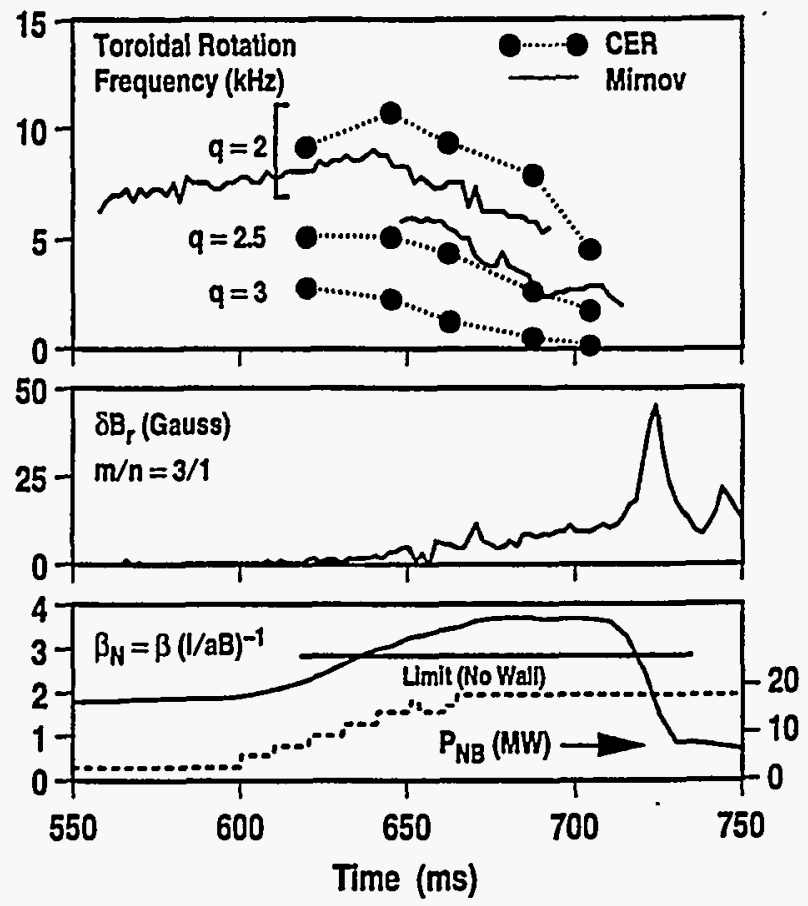

Fig. 5. The plasma $\beta$ is above the theoretical limit for no conducting wall as long as the plasma continues to rotate. When the rotation at the $q=3$ surface goes to zero, a high amplitude burst of $m / n=3 / 1$ instability activity is seen, and the plasma pressure collapses.

Among the key issues for advanced tokamak mode operation of a reactor is maintaining the core plasma rotation, particularly in RF-heated plasmas; for VH-mode operation, for wall stabilization, and particularly for avoidance of locked modes, which become more likely as the plasma size increases. Development of a method for control of the rotation profile in a reactor would be particularly valuable.

\section{Current and q Profile}

The profile most accessible for external control is the toroidal current, or the safety factor, q. The capability for some level of current profile control is a direct consequence of the need for external, noninductive current drive to maintain true steady-state operation. Current drive with a combination of neutral beams and a variety of RF waves automatically provides the capability for changing the current profile. However, in advanced tokamak operation the bootstrap current is expected to provide a significant fraction of the total current. This is beneficial because it reduces the power requirement and the need for high efficiency in the external current drive sources. The presence of a large bootstrap fraction can pose a problem unless the bootstrap profile is aligned with the desired profile since the bootstrap current is not directly controllable. Another important general consideration with noninductive current drive is that the efficiency improves as the plasma density is reduced. In DIII-D, control of the density to improve RF coupling and current drive efficiency by efficient pumping of the plasma exhaust is a major feature of the new Radiative Divertor now under construction.

The simplest characterization of the current profile is the internal inductance $\left(\ell_{j}\right)$ which measures the peakedness of the current.profile. ${ }^{d}$ Both experiment and theory show that the $\beta$ limit increases with $\ell_{\mathbf{i}}$. The DIII-D experimental results (with a few notable exceptions - e.g., rapidly rotating plasmas) are consistent with limit at $\beta_{\text {Nmax }} \approx 4 \ell_{\mathrm{i}}$ (Fig. 6). ${ }^{15}$ Confinement also improves with $\ell_{\mathrm{i}}$, as shown by the current and elongation ramp experiments (Fig. 7). The elongation ramp experiments (dK/dt $4 / s$ ) are notable because $\ell_{\mathfrak{1}}$ can be increased substantially without negative edge current. In these discharges, the confinement in ELMing $\mathrm{H}$-mode increases approximately linearly with $\ell_{\mathrm{i}}$, to $\mathrm{H} \approx 3$ at $\ell_{\mathrm{i}} \approx 1.7$ (when $\mathrm{K}$ is ramped to 2.1). ${ }^{16}$

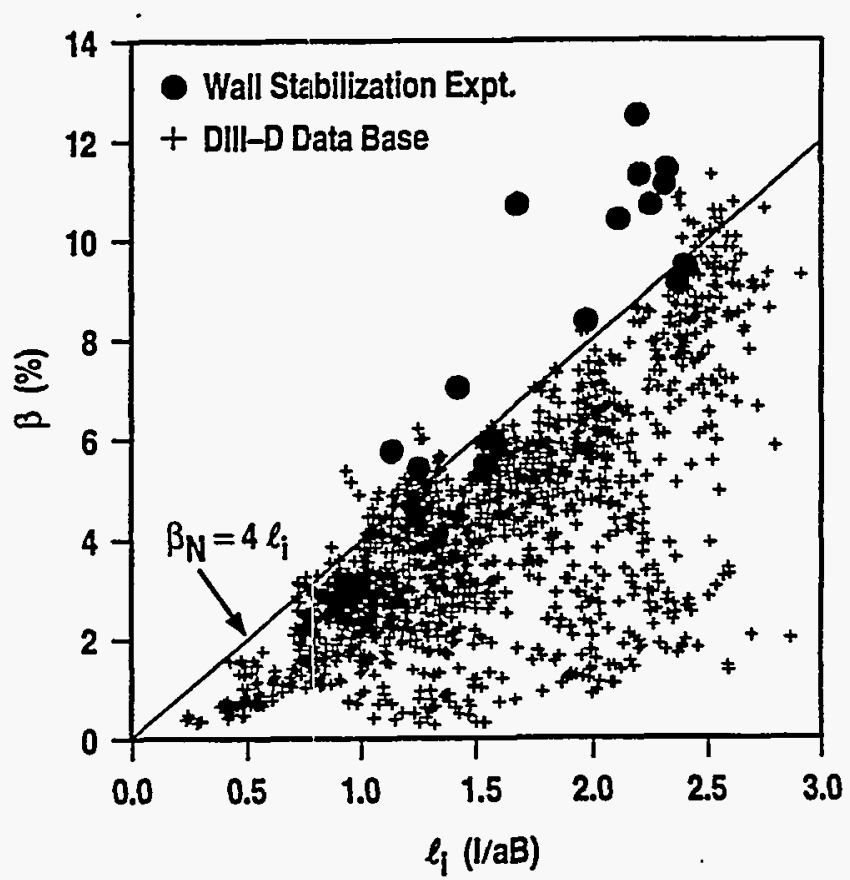

Fig. 6. The DIII-D high $\beta$ database indicates that the best achievable $\beta$ increases approximately linearly with $\ell_{\mathrm{i}}$, $\beta_{\max } \approx 4 \ell_{\mathrm{i}} \mathrm{I} / \mathrm{aB}$. Recent' experiments using wall stabilization (shown as solid dot) have been able to exceed this level.

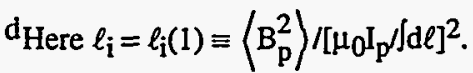




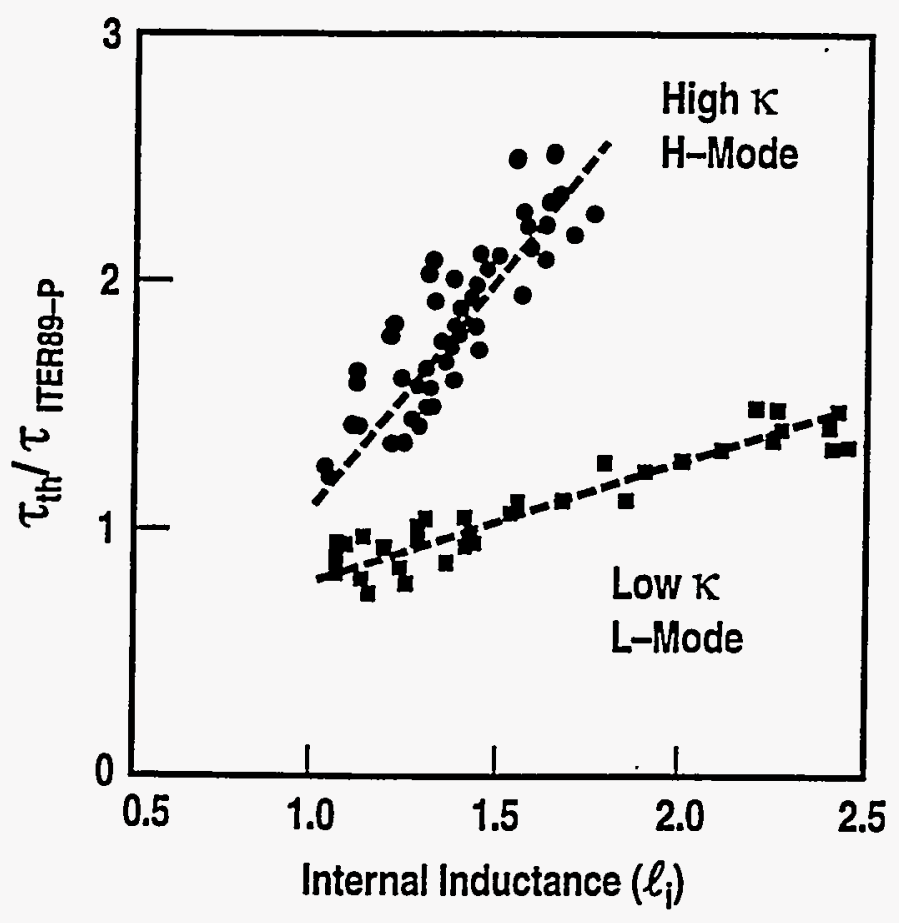

Fig. 7. Energy confinement, relative to ITER-89P scaling, increases linearly with internal inductance. Circles: data obtained with elongation ramps in $\mathrm{H}$-mode; squares: data obtained with current ramps in low elongation, $\mathrm{L}$-mode discharges.

The presence of the edge current associated with the high edge pressure gradient found in VH-mode is not optimum for stability and confinement. Use of slow current ramps $\left(\mathrm{dI}_{\mathrm{p}} / \mathrm{dt} \approx-0.5 \mathrm{MA} / \mathrm{s}\right.$; increasing $\ell_{\mathrm{i}}$ slightly, from 1.3 to 1.4 ) to reduce edge current yields an increase in $H(3.2$ to 3.9$)$ and in $\beta_{N}(3.0$ to 3.5$) .17$ Slightly faster current ramps gave less improvement or a decrease in performance. With $\mathrm{dI}_{\mathrm{p}} / \mathrm{dt}=-1.0 \mathrm{MA} / \mathrm{s}, \mathrm{VH}$-mode did not occur. In $\mathrm{H}$-mode, similar behavior is seen: a moderate decrease in edge current with a slow current ramp leads to higher internal inductance, and a significant increase in both $\mathrm{H}$ and $\beta_{\mathrm{N}}$. A more rapid current rampdown leads to loss of the edge pressure pedestal.

Negative magnetic shear $(\mathrm{dq} / \mathrm{dr}<0)$ in the central region of the plasma has provided very high $\beta$ operation of DIII-D. With high power neutral beam heating and ramping the plasma elongation to $\kappa=2.5$, a reversed shear configuration was produced which resulted in the plasma core being in the second stable ballooning mode regime. ${ }^{6}$ This allowed the pressure to rise to $\beta(0)=44 \%$ and $\langle\beta\rangle=$ $11 \%$. More recently, strongly reversed shear configurations have been produced with $q(0) / q_{\min } \geq 4$, with $\mathrm{H} \approx 3$ and $T_{i}(0)$ close to $20 \mathrm{keV}$. These experiments show that having a double valued $q$ profile with a low order rational $q$ occurring twice (even $q=1$ ) does not cause serious difficulty, and that elimination of the first regime ballooning stability limit is correlated with improvement in confinement - allowing peaking of the central pressure.

Even without reversal of the $q^{\prime}$ profile, increasing the value of $q(0)$ has been found to have beneficial effects. Low $I_{p}$, high $\beta_{p}$ experiments on DIII-D have shown global access to the second stability regime as well as improved confinement in the plasma core when $q(0)>2$ (Fig. 8). $I_{p}$ in these plasmas is predominantly bootstrap current (up to $80 \%$ ), with significant neutral beam driven current as well. ${ }^{5}$ Because both the bootstrap and neutral beam driven currents are peaked off-axis, the plasma evolves on the resistive time scale to a high $q(0)$ regime (Fig. 9). These discharges are stable to low $\mathrm{m} / \mathrm{n}$ modes, and show peaking of the plasma pressure in the core by more than a factor of 2 . However, they also show Alfven eigenmode activity which affects the fast ion confinement and thus the heating power input.

Both the reversed shear configuration and the high $\beta_{\mathrm{p}}$ plasmas have large pressure gradient, and consequently

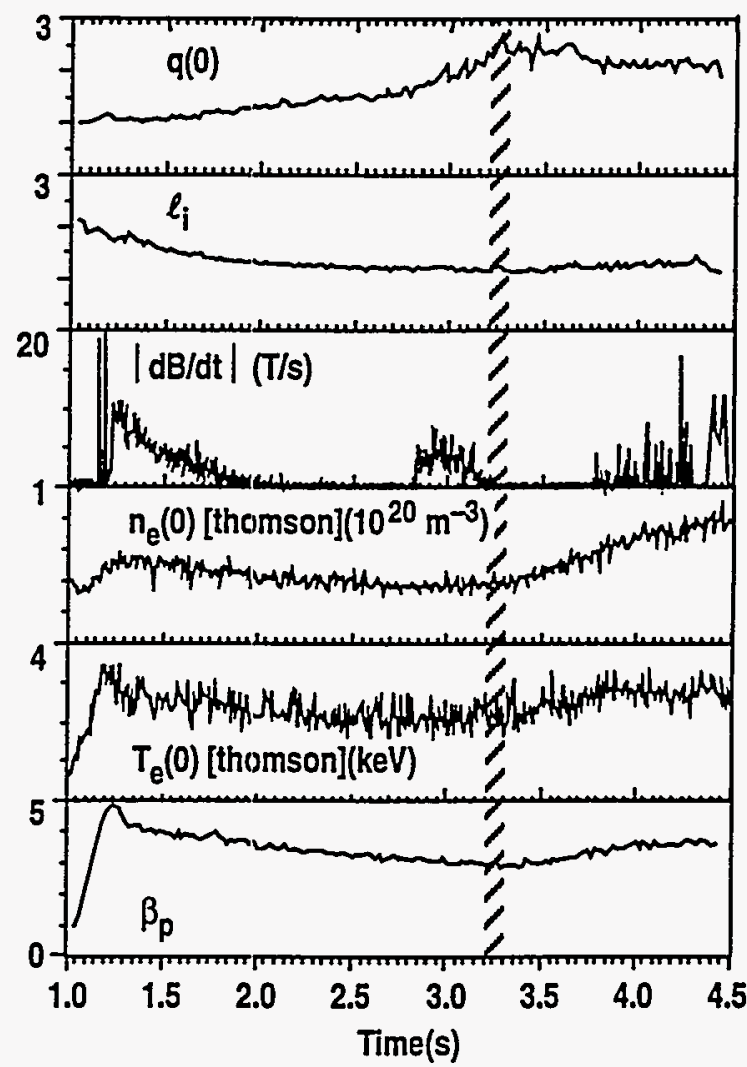

Fig. 8. Time traces showing the improvement in confinement in the plasma core when $q(0)>2$ in a low current, high $\beta_{p}$ discharge. At 3.3 seconds, $q(0) \geq 2$, low frequency Mirnov activity disappears, and both the central density and electron temperature begin to rise. 

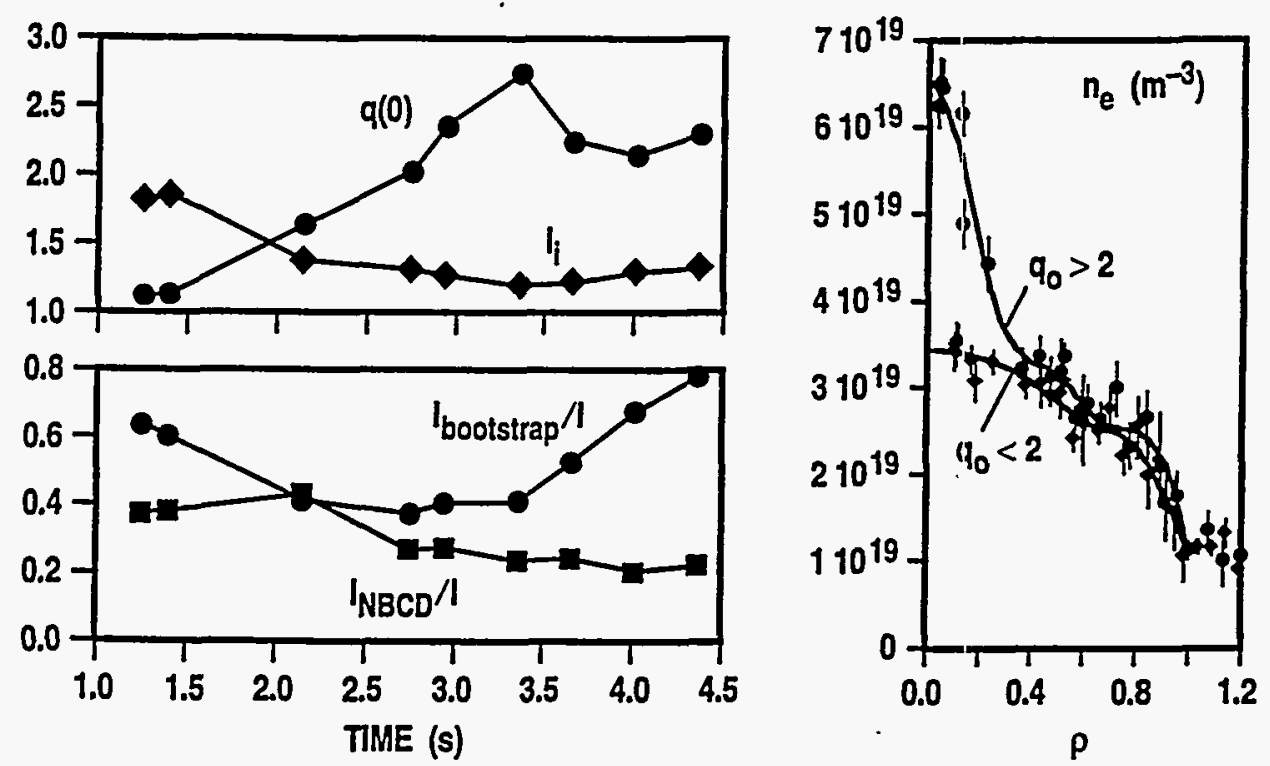

Fig. 9. Time evolution of the current profile (indicated by $q(0)$ and $\ell_{i}$ ) and the noninductive current fractions (due to bootstrap and neutral beam driven currents) for a high $\beta_{p}$ discharge. Also is the central peaking in $n_{e}$ after $q(0)$ rises above 2.0.

large bootstrap current in the inner part of the plasma profile. The resulting broadening of the current distribution in the core provides the reduction or reversal of magnetic shear which, in turn, removes the ballooning stability limits to $\mathrm{p}^{\prime}$. A key question for application of this attractive configuration to AT reactors is whether this region of improved stability and confinement can be enlarged to include a large fraction of the plasma volume. This requires moving the location of the minimum $q$ surface closer to the plasma edge.

\section{POSSIBLE ADVANCED TOKAMAK MODES}

The high $\ell_{\mathrm{i}}$ operating mode relies on a peaked plasma current profile to provide strong shear in the outer part of the plasma. This supports a large pressure gradient and allows attainment of high values of $\beta_{N}$. However, in a steady-state scenario the large edge pressure gradient, enhanced by the $\mathrm{H}$-mode transport barrier, leads to a large bootstrap current at the plasma edge. This in turn causes a broadening of the overall current profile, a reduction in $\ell_{\mathrm{i}}$, and a decrease in the stability limit. Thus, in spite of the improvements in performance seen in the experiments, no consistent high $\ell_{\mathrm{i}}$ steady-state scenario has yet been developed for DIII-D. The best we have done so far, with central current drive, is $\beta_{\mathrm{N}}=5$ at $\ell_{\mathrm{i}} \approx 1.2-1.4$ and q95 $_{95}=8$. These values have been obtained in DIII-D experiments. High confinement under these conditions is yet to be verified. The key issue for the further progress with this scenario is the development of a technique for maintaining a very low pressure gradient at the plasma edge.
As noted, the high $\beta_{\mathrm{p}}$ and the reversed central shear scenarios have similar behavior with respect to ballooning stability and peaking of the pressure profile. The difference lies in the trade-off between high $\beta$ and high $f_{b s}$. Note that $f_{b s} \propto \beta_{p}$, and that $\beta_{N} \propto\left(\beta \beta_{p}\right)^{1 / 2}$. Thus, for operation near a given stability limit $\left(\beta_{N} \leq \beta_{N \max }\right), \beta$ and $\mathrm{f}_{\mathrm{bs}}$ are inversely related. The optimization of this design choice, between higher $\beta$ - reducing the required $B$, and higher $\mathrm{f}_{\mathrm{bs}}$ - reducing the recirculating power, must be done in the context of an overall reactor design. With an increase in the region of low shear in the high $\beta_{\mathrm{p}}$ mode (in order to have second stability access), the likelihood of infernal mode instability increases (low $\mathrm{m} / \mathrm{n} \mathrm{kink}$ / ballooning modes). There are two possible ways of dealing with these modes: either they can be stabilized by making $\mathrm{q}^{\prime}<0$ rather than only $\approx 0$, or the region of low shear can be held at a value of $q$ just above 2.0 , so that the dangerous $\mathrm{m} / \mathrm{n}=5 / 2$ and $3 / 1$ surfaces are in the higher shear region toward the outside of the plasma.

Increasing the reversal of the magnetic shear in the plasma core, and exchanging bootstrap current for $\beta$, leads to the second stable core configuration, which we believe can be combined with the confinement improvement found in the VH-mode. The VH-mode provides sufficient confinement for the desired high performance regimes, $\mathrm{H}>3$. The $\mathrm{VH}$-mode is most readily obtained, and shows the greatest confinement improvement in just those strongly shaped plasmas that are suitable for improved stability. In addition, the rapid plasma rotation associated with the VH-mode is consistent with the 
requirements for rotation set by the resistive wall stabilization criteria.

\section{A SECOND-STABLE CORE, VH-MODE MODEL FOR DII-D}

The scenario we have chosen to model in detail, and to work toward in the experimental program combines the features of the reversed magnetic shear, second-stable core operating mode with the VH-mode (Fig. 10). ${ }^{19}$ The approach taken is to select a desirable $q$ profile, and through iteration, to make the current profile and the pressure profile consistent with the heating and current drive sources, while at the same time maximizing the achievable stable $\beta$. We use a simple model thermal diffusivity, which is consistent with $\mathrm{VH}$-mode observations. In the absence of a good particle transport model, an $\mathrm{H}$-modelike density profile is assumed. The DIII-D tokamak geometry and the configuration of the DIII-D heating and current drive systems is used throughout.

The stability limit is raised by increasing the central safety factor to $q(0) \approx 3.9$, while setting $q_{\min } \approx 2.6$ at $\rho \approx$ 0.6. This $q$ profile has several attractive features. Low

$$
\begin{aligned}
\beta_{N} & =5.7 \\
H & =3.5 \\
f_{b s} & =65 \% \\
\beta_{T} & =7.5 \% \\
\beta_{P} & =2.8
\end{aligned}
$$
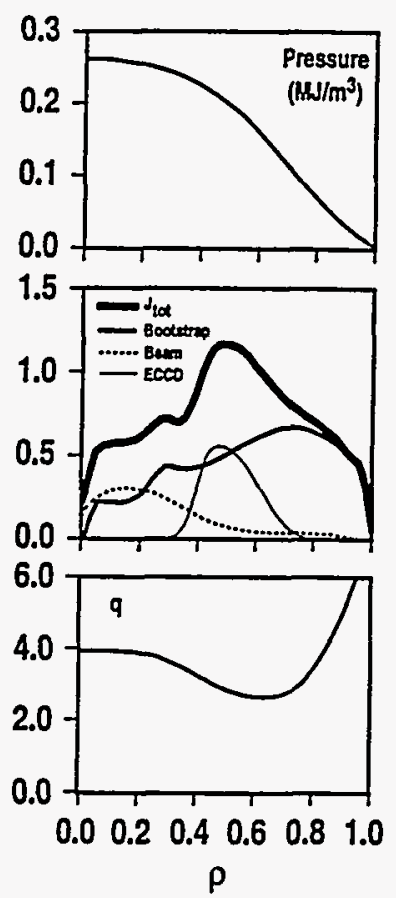

order rational surfaces are not found in the plasma. The $\mathrm{m} / \mathrm{n}=1 / 1,3 / 2,2 / 1$, and $5 / 2$ modes, which readily become unstable and can be dangerous or catastrophic for confinement, are eliminated entirely. The next most serious higher order modes, $\mathrm{m} / \mathrm{n}=3 / 1$ for example, are stabilized by being located in a region of strong shear (either positive or negative). Also, the rotation shear between the inner and outer $q=3$ surfaces helps to stabilize the resistive instability.

The analysis is done for a fixed plasma shape: $R=$ $1.68 \mathrm{~m}, \mathrm{a}=0.62 \mathrm{~m}, \mathrm{\kappa}=2.1$, and $\delta=0.8$, with good elongation and high triangularity. The magnetic field is $\mathrm{B}_{\mathrm{T}}=1.95 \mathrm{~T}$, and the plasma current is $1.6 \mathrm{MA}$. Heating and current drive are done with $60 \mathrm{MHz} \mathrm{ICH}$ (polarized and phased for fast wave current drive), $110 \mathrm{GHz} \mathrm{ECH}$, and $90 \mathrm{keV}$ neutral beams. For the final scenario, the power levels from these sources are $6.5 \mathrm{MW}, 7.0 \mathrm{MW}$, and $6.5 \mathrm{MW}$, respectively. In order to move the minimum of the q profile well away from the axis, off-axis current drive is required. The ECCD is used for this, with the location being adjusted by moving the resonance vertically, as it will be done in DIII-D with a movable mirror.

$$
\begin{aligned}
I_{p} & =1.6 \mathrm{MA} \\
B_{T} & =1.95 \mathrm{~T} \\
\kappa & =2.1 \\
\delta & =0.8 \\
q_{95} & =6.5 \\
P_{\text {TOT }} & =20.0 \mathrm{MW} \\
\mathrm{P}_{\mathrm{ECH}} & =7.0 \mathrm{MW} \\
\mathrm{P}_{\mathrm{FW}} & =6.5 \mathrm{MW} \\
\mathrm{P}_{\mathrm{NBI}} & =6.5 \mathrm{MW}
\end{aligned}
$$

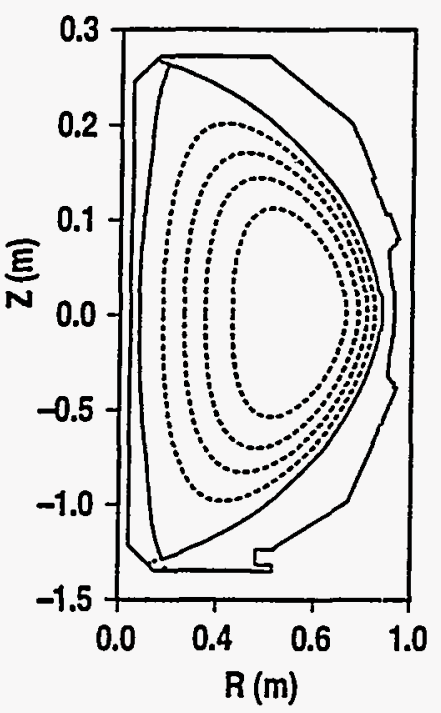

Fig. 10. A summary of the second-stable-core VH-mode scenario. Shown are the pressure profile, the current profile and its components ( $\mathrm{J}_{\text {bootstrap }} \mathrm{J}_{\mathrm{beam}}$, and $\mathrm{J}_{\mathrm{ECCD}}$ ), the $\mathrm{q}$ profile, and the flux surface contours. 
The coupled heating, current drive, transport, and equilibrium calculations are carried out using the ONETWO ${ }^{19}$ code. In this code, the rf deposition and current density profiles are calculated for a given equilibrium using the auxiliary codes FASTWAVE ${ }^{20}$ for the ICRF and TORAY ${ }^{21}$ for the ECRF power. Using a selected transport model, recalculating the equilibrium as needed, time is advanced until a stationary state is reached. The electron thermal diffusivity is taken to be the INTOR model: $\mathrm{n}_{\mathrm{e}} \chi_{\mathrm{e}}=5 \times 10^{19} /\left(1-0.8 \mathrm{p}^{2}\right) \mathrm{m}^{-1} \mathrm{~s}^{-1}$, and the ion diffusivity is a multiple of neoclassical scaling: $\chi_{i}=$ $2.6 \chi_{i}^{\text {neo }}$. The coefficients here are adjusted to give a global thermal energy confinement time $\tau_{E}=$

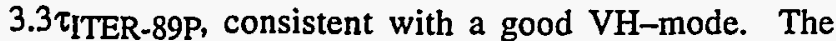
density profile is representative of VH-mode, and is fixed throughout the simulation. The line-averaged density is $5.7 \times 10^{19} \mathrm{~m}^{-3}$, and the density at the boundary is $2 \times 10^{19} \mathrm{~m}^{-3}$.

The temperature profiles show a broad, rather flat region at $\rho \leq 0.4$. This results from the significant offaxis power input to the electrons from the $\mathrm{ECH}$, and from the large value of the ion thermal conductivity near the axis, which in turn is due to the increase in $\chi_{i}^{\text {neo }}$ as $q$ rises near the axis.

The self consistent current profile obtained with ONETWO is very close to the target profile. Of the total current of 1.6 MA, 1.07 MA is bootstrap current (66\%), $0.17 \mathrm{MA}$ is neutral beam driven current, and $0.32 \mathrm{MA}$ is ECCD. In the example shown, the localized current minimum at the axis leads to a narrow peak in $q$ with $q(0) \sim 4-5$. This can be controlled with a small amount of FWCD. This equilibrium is calculated to be stable to $n=1$, $n=2$, and ballooning modes at $\beta_{N}=5.5$, with $\beta=7.1 \%$.

Further stability analyses have been done on model equilibria based on the one generated with ONETWO, in order to look at the variation of the profiles with changing pressure, and at the stabilizing effect of the DIII-D wall. The low $\mathrm{n}$ mode ideal MHD stability is calculated using the code GATO,22 and the ballooning stability is calculated with the CAMINO ${ }^{23}$ code. A series of cases were calculated, with increasing $\beta$. As $\beta_{\mathrm{N}}$ increased from 2.4 to 6.5 , the details of the current and $q$ profiles changed. In particular, the current profile broadened, with $\ell_{\mathrm{i}}$ decreasing from 0.74 to 0.55 , and the edge current density increased with $\mathrm{J}_{95} /\langle\mathrm{J}\rangle$ going from 0.23 to 0.64 . $^{\mathrm{e}}$

$\mathrm{e}_{\mathrm{J}_{95}}$ is the flux surface averaged current density at the $95 \%$ flux surface, and $\langle\mathrm{J}\rangle$ is the average current density over the entire plasma cross-section.
The dependence of the low $\mathbf{n}$ stability limit on the location of the wall is analyzed using a wall that has the shape of the actual DIII-D vessel. For the double-null plasma used here, this corresponds approximately to $r_{w a l l} / a=1.3$. At this location the plasma is stable to $n=1$ for $\beta_{N}>6$, and to $n=2$ modes for $\beta_{N} \geq 5.7$. If the wall dimensions are doubled, keeping the plasma the same, the limiting $\beta_{N}$ drops to 3.2 , and with no wall, the stability limit is at $\beta_{N}=2.8$ (Fig. 11).

\section{DISCUSSION AND CONCLUSIONS}

In order to provide experimental verification of the existence and accessibility of advanced tokamak operating modes in DIII-D, we have identified at least one internally consistent scenario that plausibly provides both high confinement and high stability limits in steady-state. This effort is based on examination of experiments which show some aspect of improved performance and an attempt to extract the key features that lead to the improvements. Among the conditions that lead to notable improvements in stability and confinement are high plasma triangularity, bulk plasma rotation (to provide stabilizing interaction with the wall), strongly sheared radial electric fields and toroidal rotation (to reduce turbulence and turbulent transport), high $q(0)$, low or reversed magnetic shear in the plasma interior, and a finite current

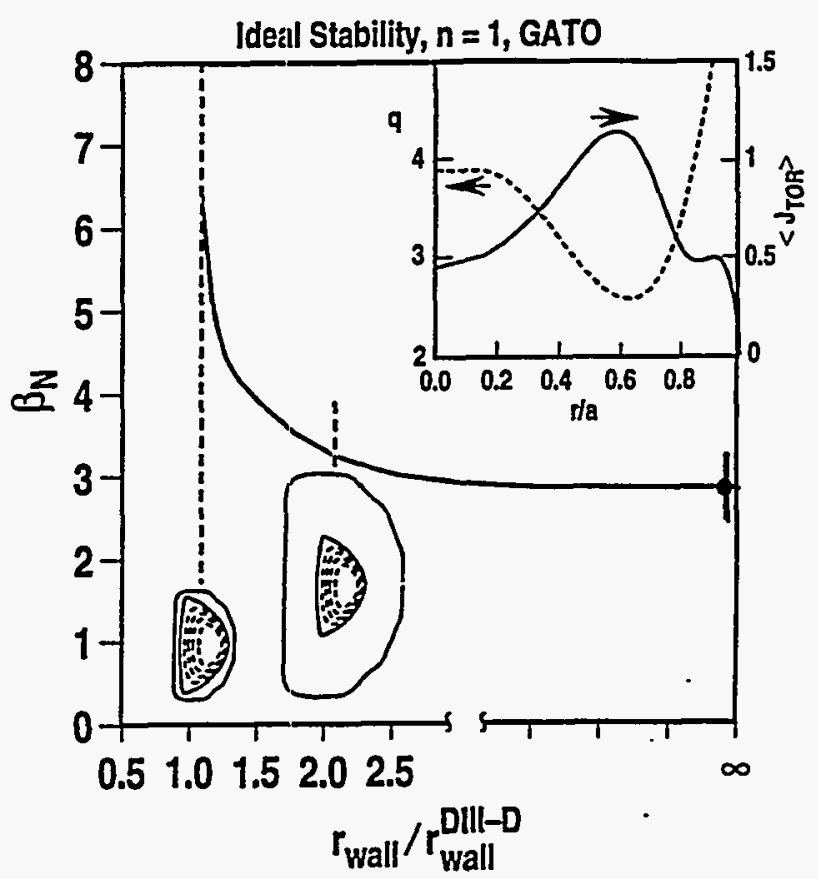

Fig. 11. The calculated stability of a pressure and current profile characteristic of the second-stable-core VH-mode plasma is greatly improved by a close resistive wall. With a resistive wall at the location of the DIII-D wall, the $\beta$ limit is roughly double that with no wall. 
density at the plasma edge. The key physics elements of these improvements are the reduction in turbulence by the $\mathrm{E}_{\mathrm{r}}$ shear and the elimination of the first regime ballooning limits, and the improved low n mode stability resulting from the triangularity, the magnetic shear, and the bulk plasma rotation. The scenario developed in detail is the second-stable core $\mathrm{VH}$-mode. It is based on combining the improved core confinement and high $\beta$ seen with reversed magnetic shear in the second-stable core experiments, the improved core confinement seen at high $q(0)$ in the high $\beta_{p}$ experiments, and the overall improvement associated with the VH-mode of operation. Development of other scenarios is under way.

The present DIII-D program is directed toward developing these advanced tokamak modes of operation. We are now completing the installation of a $6 \mathrm{MW} \mathrm{ICH}$ system for heating and fast wave current drive. We expect to install $2 \mathrm{MW}$ of $110 \mathrm{GHz} \mathrm{ECH}$ next year, with the capacity of the ECH system increasing to $10 \mathrm{MW}$ by 1998. In the coming year the DIII-D advanced tokamak research program will emphasize assessment of the roles of magnetic shear, $E_{r}$ shear, and plasma rotation in improved confinement and stability, initial studies of feedback control of the current profile, and work toward demonstration of a fully noninductive, fast wave current drive plasma.

The question of heat and particle removal has not been discussed in this paper. However, we note that the high triangularity, double-null shape appropriate for the second-stable core $\mathrm{VH}$-mode may provide some challenges to the designers of divertors. In particular, the $\mathrm{X}$-points are at small major radius, reducing the space available for heat removal and pumping, and the distance between the X-point and the wall along the inner leg of the separatrix is short. The Radiative Divertor now being designed for DIII-D will have this geometry, and will test the consistency between the AT plasma configuration and effective divertor operation.

\section{ACKNOWLEDGMENT}

This is a report of work supported by the U.S. Department of Energy under Contract Nos. DE-AC0389ER51114, DE-AC05-84OR21400, and W-7405-ENG48.

\section{REFERENCES}

${ }^{1}$ J.D. Galambos, Oak Ridge National Laboratory Report ORNL/TN12483, submitted to Nucl. Fusion.
2 M. Kikuchi, et al., Plasma Phys. and Contr. Nucl. Fusion Research (Würzburg, 1992), IAEA, Vienna, Vol. 1, p. 189; S. Ishida, et al., ibid., Vol. 1, p . 219; JET Team, ibid., Vol. 1, p. 329; M. Mauel, et al., ibid., Vol. 1, p. 205; M. Zarnstorff, et al., ibid., Vol. 1, p. 111; T.S. Taylor, et al., ibid., Vol. 1, p. 167; JT-60 Team, Plasma Phys. and Contr. Nucl. Fusion Research (Seville, 1994), IAEA, Vienna, to be published; Y. Koide, et al., ibid; Y. Kamada, et al., ibid.

${ }^{3}$ G.L. Jackson, et al., Phys. Rev. Lett. 67, 3098 (1991); C.M. Greenfield, et al., Plasma Phys. and Contr. Fusion 35 (1993) B263.

${ }^{4}$ L.L. Lao, et al., Phys. Rev. Lett. 70, 3435 (1993); L.L. Lao, et al., Plasma Phys. and Contr. Nucl. Fusion Research (, 1992), IAEA, Vienna, Vol. 1, p. 563; J.R. Ferron, et al., Phys. Fluids B 52532 (1993).

5P.A. Politzer, et al., Phys. Plasmas 1, 1545 (1994).

${ }^{6}$ E.A. Lazarus, et al., Phys. Fluids B 3, 2220 (1991); E.A. Lazarus, et al., Phys. Fluids B 4, 3644 (1992).

7 T.S. Taylor, et al., Plasma Phys. and Contr. Nucl. Fusion Research (Würzburg, 1992), IAEA, Vienna, Vol. 1, p. 167; E.A. Lazarus, et al., Plasma Phys. and Contr. Nucl. Fusion Research (Seville, 1994), IAEA, Vienna, to be published; T.H. Osborne, et al., Plasma Phys. and Contr. Fusion 36 (1994) A237.

${ }^{8}$ K.H. Burrell, et al., Plasma Phys. and Contr. Fusion 36 (1994) A291; J. Kim, et al., ibid., A183.

${ }^{9}$ T.H. Osborne, et al., Plasma Phys. and Contr. Fusion 36 (1994) A237.

${ }^{10}$ R.D. Stambaugh, et al., Plasma Phys. and Contr. Nucl. Fusion Research (Seville, 1994), IAEA, Vienna, to be published.

${ }^{11}$ R.J. La Haye, et al., General Atomics Report GAA21544 (1993), submitted to Phys. Rev. Lett.; K.H. Burrell, et al., Phys. Plasmas 1, 1536 (1994).

${ }^{12}$ A. Bondeson and D.J. Ward, Phys. Rev. Lett. 72, 2709 (1994); T.H. Jensen and M.S. Chu, J. Plasma Physics 30, 57 (1983).

${ }^{13}$ E.J. Strait, et al., Proc. of the 21st European Physical Society Conference on Controlled Fusion and Plasma Physics (1994). 
${ }^{14}$ T.S. Taylor, et al., "Wall Stabilization of High Beta Plasmas in DIII-D," to be published in Phys. of Plasmas; General Atomics Report GA-A21914 (1994).

${ }^{15}$ E.J. Strait, Phys. Plasmas 1, 1415 (1994).

${ }^{16}$ L.L. Lao, et al., Phys. Rev. Lett. 70, 3435 (1993).

17J.R. Ferron, et al., Phys. Fluids B 5, 2532 (1993).

${ }^{18}$ T.S. Taylor, et al., "Optimized Profiles for Improved Confinement and Stability in the DIII-D Tokamak," in Proc. 21st Euro. Conf. on Plasma Physics and Contr. Fusion, Montpellier France, 1994, to be published.
${ }^{19}$ H. St. John, et al., "Coupled MHD and Transport Analysis of Improved Confinement DIII-D Discharges," in Proc. 20st Euro. Conf. on Plasma Physics and Contr. Fusion, Lisbon Portugal, 1993, (European Physical Society, Petit-Lancy, Switzerland, 1994), Vol. 1, p. 99.

${ }^{20}$ S.C. Chiu, et al., Nucl. Fusion 29, 2175 (1989).

${ }^{21} \mathrm{~K}$. Matsuda, IEEE Trans. on Plasma Science 17, 6 (1989).

${ }^{22}$ L.C. Bernard, et al., Comput. Phys. Commun. 24, 377 (1981)..

${ }^{23}$ M.S. Chance, in "Theory of Fusion Plasmas," Proc. of the Varenna Workshop 1987 (Editrice Compositori, Bologna, 1987). 